\title{
Using pictograms improves the understanding of Stock-Flow Systems
}

\author{
Friederike Brockhaus ${ }^{1}$ and Peter Sedlmeier ${ }^{1}$ \\ ${ }^{1}$ Chemnitz University of Technology, Institut of psychology, \\ 09107 Chemnitz, Germany \\ \{friederike.brockhaus, peter.sedlmeier\}@psychologie.tu-chemnitz.de
}

\begin{abstract}
We examined if we could improve performance in so-called StockFlow Systems (SF-systems). SF-systems are dynamic systems, which consist of a stock and two flows - the inflow and the outflow. The stock changes depending on the flows' patterns. Many persons have problems understanding these systems (Sterman, 2002). Up to now, only one modification of the flows' representation format led to rather strongly enhanced SF-performance: using pictograms instead of line graphs (Brockhaus, Arnold, Schwarz, \& Sedlmeier, 2013). The present experiment analyzed if pictograms led to better performance when the SF-task was somewhat changed; this time, we focused on basic SF-understanding. Additionally, it analyzed, if further modifications of the diagram - emphasizing the distinction of the two flows and emphasizing the calculated difference of inflow minus outflow - could improve SFperformance. Results showed, the effect was smaller, and participants working on pictograms only solved about $50 \%$ or less of the tasks correctly. However, using pictograms improved SF-performance again.
\end{abstract}

Keywords: stock-flow systems / representation format / pictograms

\section{Introduction}

We wanted to find out if modifications of the representation of the flows could improve performance in SF-Systems. SF-Systems consist of an inflow, an outflow and a stock, which changes in dependence of the in- and outflow. Examples for SFSystems may be your bank account with money as the stock or the atmosphere with $\mathrm{CO}_{2}$ concentrations as the stock. In your bank account, the inflow can be your salary, the outflow your expenditures and the stock is the balance, which changes due to the incoming and outgoing money. It is important to understand SF-systems themselves, since they are omnipresent in our personal life, in society, in business, in nature etc. Furthermore, SF-Systems are part of complex dynamic systems, which are very difficult to understand, e.g. the worldwide climate. Often, it was assumed, that these difficulties stem from the high amount of parts or building blocks, complex dynamic systems consist of (Brehmer, 1992). However, the building blocks themselves often are difficult to understand, as exponential growth or SF-systems. 
Therefore, understanding these parts of complex systems is preliminary to understand the systems' complexity. Nevertheless, when people have to understand or manage SF-systems, they often fail even if they are highly educated. They often violate the "principle of accumulation": They do not realize that as long as the inflow is exceeding the outflow, the stock is increasing and vice versa (Cronin, Gonzalez, \& Sterman, 2009).

First authors to analyze SF-Systems were Booth Sweeney and Sterman (2000). In their study about SF-Systems, they created among others a task about a tub of water. In that task, participants received a graph of the in- and outflow (Fig. 1).
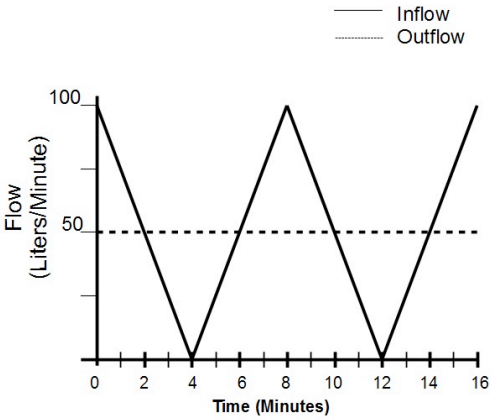

Fig. 1. Triangle task (modified from Booth Sweeney \& Sterman, 2000; taken from (Brockhaus et al., 2013)

Then they were to judge the change of the stock (water in the tub). They had to draw the changes of the stock over time into an empty diagram. In somewhat modified studies, participants had to answer questions about the changes of the stock. Many participants were incapable to solve the SF-tasks correctly. Often $40 \%$ up to $70 \%$ and more of the participants committed errors (Booth Sweeney \& Sterman, 2000; Cronin et al., 2009; Ossimitz, 2002).

Many modifications did not or only slightly improve SF-understanding (Cronin et al., 2009; Roeder, 2017; Schwarz, 2016). Following authors who claim, a graph can be more or less helpfull in dependence of its design (Macdonald-Ross, 1977), (Brockhaus et al., 2013) changed the often used line graphs into a kind of pictorial chart similar to those proposed by (Neurath, 1936). Indeed, the changed representation format of the flow graphs led to better SF-performance: $57 \%$ to $83 \%$ correctly solved the tasks including pictograms whereas in the baseline condition with line graphs only $23 \%$ to $30 \%$ of the participants correctly solved the task. These were large and significant effects. One possible drawback was the fact, that participants calculated the stock for each moment. 


\section{Present study}

In the present study, we changed the tasks wanted to find out if the surprisingly good performance still occurred in the modified tasks. Participants now answered questions instead of drawing the stock. In that way, only basic SF-knowledge is necessary and the participants might be less invited to calculate the stock's behavior for each moment. We designed the tasks so that the participants could easily infer the stock's maximum and minimum in case they understood the principle of accumulation. Furthermore, we modified the pictograms, iconic signs were used that showed directly the difference of in- and outflow (for example birth rates represent the inflow and death rates the outflow, babies and coffins are used instead of stick figures for all). The latter modification referred to ISOTYPE, which can be seen as a picture language and includes advices how to create pictorial charts (Neurath, 1936). We also modified the diagrams, so that the calculated difference of in- and outflow could be seen, that means the conditions including pictograms or iconic signs were presented either with or without emphasizing if the inflow exceeds the outflow or vice versa.

Our main hypothesis was that participants working with pictograms or iconic signs do better than those working with line graphs do. We additionally wanted to find out if iconic signs work better than pictograms and if emphasizing the calculated difference of in- and outflow is helpful.

\subsection{Method}

149 students of Chemnitz University of Technology (mean age = 22 years, $S D=3.8$; range 18 to 24 years; 97 Students of psychology (65\%) and 48 students of Sensors and Cognitive Psychology; 32 male (21\%) and 113 female ${ }^{1}$ ) took part. The participants solved three tasks of different flow patterns (one as in Fig. 1, one as in Fig. 2, and a third as in Fig. 3 ) in different sequences.

\footnotetext{
${ }^{1}$ Some participants did not give that information
} 


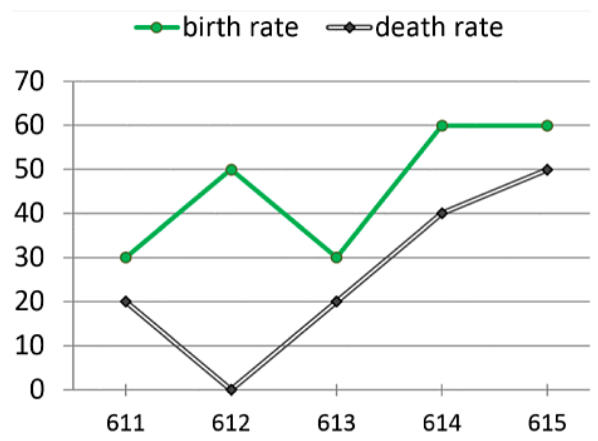

Fig. 2. One SF-task with flows shown as line graph (patterns of flows modified from Cronin et al., 2009).

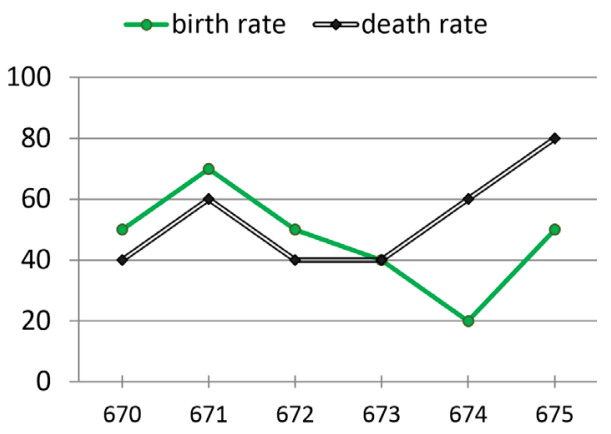

Fig. 3. SF-task with flows shown as line graph (patterns of flows slightly modified from Cronin et al., 2009).

The task's topic was the population of a village, changing in dependence of birth and death rates. Each participant solved all three tasks in one of five different conditions:

(1) baseline with line graphs (Fig. 1 to Fig. 3)

(2) pictograms with identical stick figures for both of the flows and no emphasis of the calculated difference (Fig. 4 (a))

(3) pictograms with emphasis (Fig. 5 (a))

(4) iconic signs with different signs for inflow and outflow and no emphasis (Fig. 4 (b))

(5) iconic signs with emphasis (Fig. 5 (b)).

To solve one task, the participant had to answer two questions: When is the stock maximal and when minimal. The answers were coded as correct ( 1 point) or wrong (0 points). To analyze SF-performance, we calculated the sum of all tasks and questions; it ranged from 0 to 6 points. 


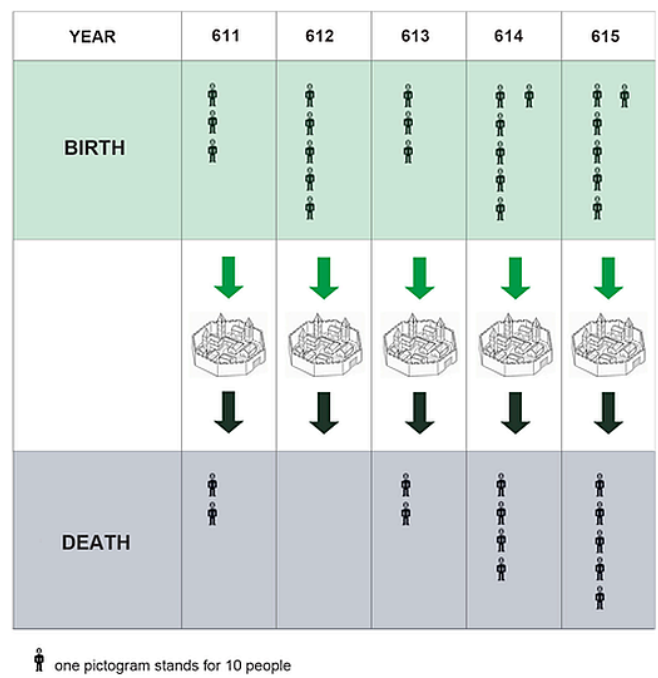

(a)

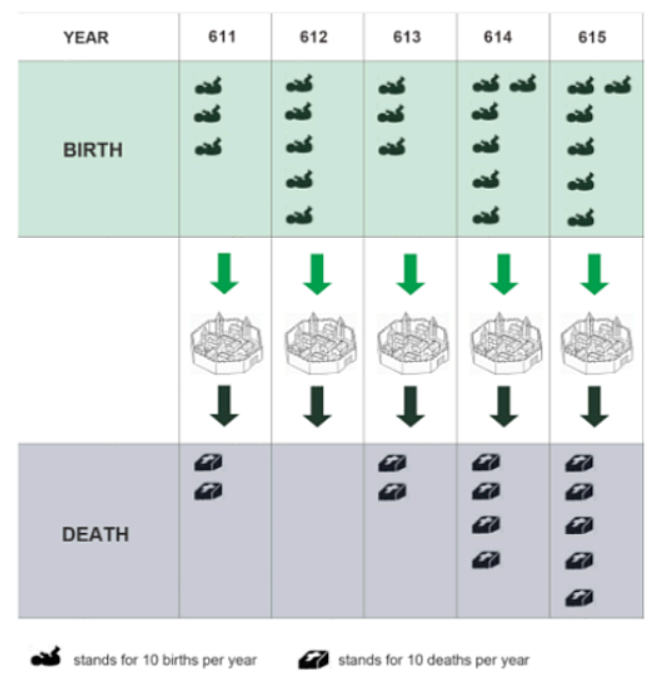

(b)

Fig. 4. Same patterns of flows as in Fig. 2, shown as pictograms (condition (2)) and iconic signs (condition (4)) (Arntz, G. "Pictograms" (Babies and coffins) / (C) VG Bild-Kunst, Bonn 2018) 


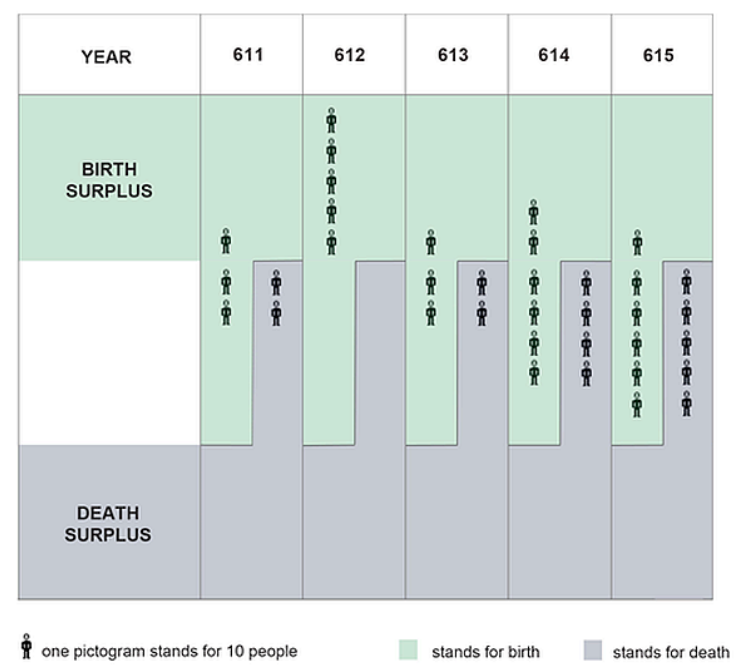

(a)

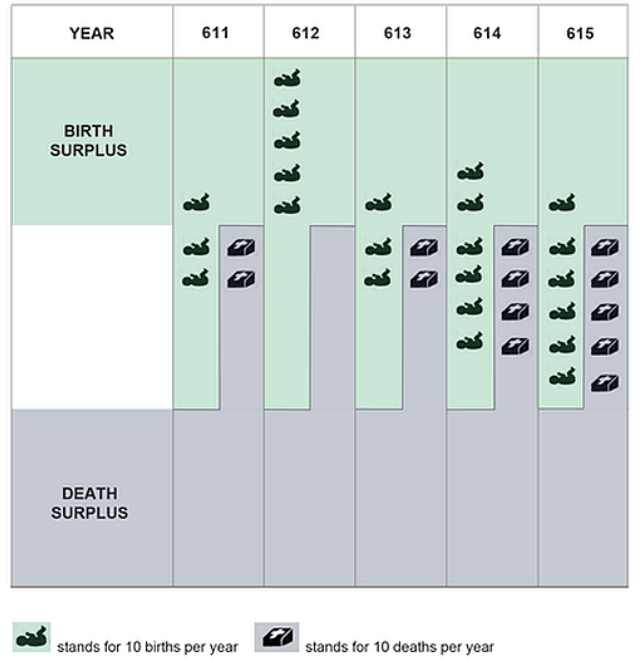

(b)

Fig. 5. Same patterns of flows as in Fig. 2, shown as pictograms and iconic signs (Arntz, G. "Pictograms" (Babies and coffins) / (C) VG Bild-Kunst, Bonn 2018), this time emphasizing the difference of in- and outflow (conditions (3) and (5)).

\subsection{Results}

Indeed, the performance was still better in the conditions with pictograms or iconic signs whereas emphasizing which flow is larger is not helpful. The mean percentage of correct solutions was for baseline $30 \%$, pictograms $48 \%$, pictograms and emphasis 
$41 \%$, iconic signs $53 \%$, iconic signs and emphasis $44 \%$. Fig. 6 shows the means of the sum for all solutions (0 to 6 points).

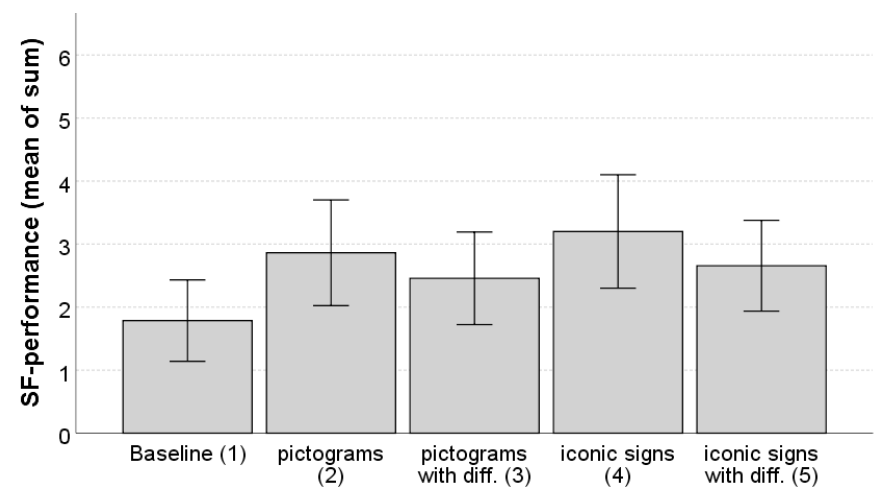

Fig. 6. Mean sum (ranging from 0 to 6 points) for SF-performance in the five conditions with $90 \%$ confidence intervals.

Regression analysis with mean sum as dependent variable and several predictors (conditions and some possible confounding variables) also revealed that SFperformance was still worse when line graphs were used: $R^{2}=.22, F(10,120)=3.424$; $p<.00^{2}$. Baseline being better than all other conditions showed $B=-.20(p=.02)$, which meant baseline significantly led to worse results; iconic signs should lead to better performance than pictograms: $B=.04(p=.33)$; emphasizing difference should lead to better performance: $B=-.08(p=.19)$; both variables did not significantly differ from zero. Further predictors were: sex, subject of study, grade of mathematics, femininity and masculinity (both measured by Bem sex role inventory revisited, (Troche \& Rammsayer, 2011) their interaction and finally a variable if participants had worked on SF-tasks in a former study. The only significant predictors were mathematical grade $(B=.26 ; p<.00$, the better the grade, the better SFperformance) and femininity scale $(B=-.27 ; p=.01$; the higher femininity value the worse SF performance) (for further information (Brockhaus, in press).

\subsection{Discussion}

Again, pictograms and this time used iconic signs led to better SF performance. But using pictograms or iconic signs did not influence SF-performance to the same extent as in the study before (Brockhaus et al., 2013). It seemed that calculating the stock for each moment increased the SF-performance, and the pictograms probably induced the correct calculations. In that study and in the present one, mainly

2 One outlier was excluded from regression analysis 
participants socialized in Germany, and so in a "Western country", and welleducated participants took part. Generalizing the results to other cultural or educational backgrounds is not possible and further studies should analyze that. However, the present study showed that iconic representation of the flows still led to better judgments than line graphs in our sample, so in the moment, the best advice is to use iconic signs when communicating information dealing with SFproblems.

\section{References}

Booth Sweeney, L., \& Sterman, J. D. (2000). Bathtub dynamics: initial results of a systems thinking inventory. System Dynamics Review, 16(4), 249-286.

Brehmer, B. (1992). Dynamic decision making: Human control of complex systems. Acta Psychologica, 81(3), 211-241.

Brockhaus, F. (in press) Von den Flussgroeßen zum Bestand: Lassen sich einfache dynamische Systeme intuitiv verstaendlich darstellen? (Doctoral Thesis). Chemnitz University of Technology, Chemnitz, Germany.

Brockhaus, F., Arnold, J., Schwarz, M., \& Sedlmeier, P. (2013). Does the modification of the representation format affect stock-flow-thinking? Proceedings of the 31st International Conference of the System Dynamics Society in Cambridge, MA, USA.

Cronin, M. A., Gonzalez, C., \& Sterman, J. D. (2009). Why Don't Well-Educated Adults Understand Accumulation? A Challenge to Researchers, Educators, and Citizens. Organizational Behavior and Human Decision Processes, 108(1), 116-130.

Macdonald-Ross, M. (1977). How numbers are shown. Educational Technology Research and Development, 25(4), 359-409.

Neurath, O. (1936). International Picture Language. . London: Kegan Paul. .

Ossimitz, G. (2002). Stock-Flow-Thinking and Reading stock-flow-related Graphs: An Empirical Investigation in Dynamic Thinking Abilities. Paper presented at the International System Dynamics Conference.

Roeder, V. (2017). The Attempt to Boost Stock Flow Performance by Using a General Problem Solving Strategy and a Reappearing Gender Effect. Paper presented at the 35th International Conference of the System Dynamics Society, Cambridge, Massachusetts, USA.

Schwarz, M. A. (2016). Vom stockenden Verständnis fließender Zusammenhänge. Darstellungs- und personenbezogene Einflussfaktoren auf das basale Verständnis einfacher dynamischer Systeme. (Doctoral Thesis). Chemnitz University of Technology, Chemnitz, Germany.

Sterman, J. D. (2002). All Models are Wrong: Reflections on Becoming a Systems Scientist. System Dynamics Review, 18, 501-531.

Troche, S., \& Rammsayer, T. H. (2011). Eine Revision des deutschsprachigen Bem Sex-Role Inventory. Klinische Diagnostik und Evaluation, 4, 262-283. 